\title{
FAUNA DO SOLO AO LONGO DO PROCESSO DE SUCESSÃO ECOLÓGICA EM VOÇOROCA REVEGETADA NO MUNICÍPIO DE PINHEIRAL - RJ
}

\author{
SOIL FAUNA ALONG THE PROCESS OF ECOLOGICAL SUCCESSION IN GULLIES \\ REVEGETATED IN THE MUNICIPALITY OF PINHEIRAL - RJ
}

\author{
Khalil de Menezes Rodrigues ${ }^{1}$ Maria Elizabeth Fernandes Correia ${ }^{2}$ Alexander Silva de Resende ${ }^{3}$ \\ Fernanda de Lima Camilo ${ }^{4}$ Eduardo Francia Carneiro Campelo ${ }^{3}$ Avílio Antônio Franco ${ }^{5}$ \\ Sonia Carmela Falci Dechen ${ }^{6}$
}

\begin{abstract}
RESUMO
O objetivo do trabalho foi avaliar a diversidade, riqueza e composição da comunidade da fauna do solo epígea em uma voçoroca revegetada no ano de $2000 \mathrm{com}$ diferentes espécies de leguminosas arbóreas. A voçoroca está localizada no Instituto Federal de Educação (IFRJ) campus Nilo Peçanha no município de Pinheiral - RJ. Foram avaliadas as seguintes áreas: plantio de Acacia mangium Willd. (PA), plantio de Mimosa caesalpiniifolia Benth. (PM), plantio de leguminosas arbóreas no terço superior da encosta (PTS), plantio de leguminosas no interior da voçoroca (IVR2000). Os plantios de A. mangium e M. caesalpiniifolia foram realizados antes da revegetação. Para efeito comparativo também foi avaliada uma área de floresta secundária com 20 anos, próxima ao local de coleta. Na coleta da fauna do solo epígea foram utilizadas 10 armadilhas do tipo pitfall (MOLDENKE, 1994) por área. Foram realizadas três coletas, uma no início da revegetação, em maio de 2000, em junho de 2006 e abril de 2008. Houve um aumento no número de grupos e na diversidade da fauna do solo no plantio no terço superior, no interior da voçoroca, e no plantio de Mimosa caesalpiniifolia após 6 anos da intervenção. Nas coletas realizadas em 2000 e 2006, a APC mostrou que a maioria dos grupos estava associada ao ano de 2006, mostrando total antagonismo com o ano de 2000. O plantio de Acacia mangium foi a área com o menor número de grupos. Na coleta realizada em 2008, a pastagem foi a área com menor número médio de grupos coletados e na análise de componentes principais (ACP) do interior da voçoroca os grupos estavam associados ao ano de 2008 com total antagonismo com os anos de 2000 e 2006. Os grupos Formicidae e Collembola (Entomobryomorpha) foram os mais dominantes em todas as áreas amostradas no ano de 2000 e 2008 e no interior da voçoroca ano de 2006.
\end{abstract}

Palavras-chave: recuperação de áreas degradadas; leguminosas arbóreas; fauna epígea.

\section{ABSTRACT}

The diversity, richness and composition of the soil epigeous fauna community were evaluated in a revegetated gully at the Instituto Federal de Educação (IFRJ) campus Nilo Peçanha, in the municipality of

1 Engenheiro Agrônomo, Dr., Professor do Centro de Ciências Agrárias e Ambientais da Universidade Federal do Maranhão, BR 222, KM 04, s/n, Boa Vista, Chapadinha - MA. CEP 65500-000, Chapadinha (MA), Brasil. agrokhalil@yahoo.com.br

2 Bióloga, Dra ., Pesquisadora do Centro Nacional de Pesquisa de Agrobiologia, BR 465, Km 7, CEP 23890-000, Seropédica (RJ), Brasil. elizabeth.correia@embrapa.br

3 Engenheiro Florestal, Dr., Pesquisador do Centro Nacional de Pesquisa de Agrobiologia, BR 465, Km 7, CEP 23890-000, Seropédica (RJ), Brasil. alexander.resende@embrapa.br; eduardo.campello@embrapa.br

4 Bióloga, MSc., Doutoranda em Biologia Animal, Universidade Federal Rural do Rio de Janeiro, BR 465, Km 7, CEP 23890-000, Seropédica (RJ), Brasil. fernandinhacamilo@gmail.com

5 Engenheiro Agrônomo, Dr., Pesquisador do Centro Nacional de Pesquisa de Agrobiologia, BR 465, Km 7, CEP 23890-000, Seropédica (RJ), Brasil. avilio.franco@embrapa.br

6 Engenheira Agrônoma, Dra ${ }^{\mathrm{a}}$, Pesquisadora do Centro de Pesquisa e Desenvolvimento de Solos e Recursos Ambientais, Instituto Agronômico, IAC. Av. Barão de Itapura, 1481, Botafogo. CEP 13020-902 Campinas (SP), Brasil. dechen@iac.sp.gov.br

Recebido para publicação em 16/08/2010 e aceito em 29/07/2014 
Pinheiral, Rio de Janeiro state. Revegetation was performed in 2000, and consisted of four different strategies involving N-fixing trees: planting Acacia mangium Willd. (PA); planting Mimosa caesalpiniifolia Benth. (PM); on the outer edges of the gully head (PTS); and planting a mix of legume tree species into the gully (IVR2000). Acacia mangium and Mimosa caesalpiniifolia were planted above the gully head. A nearby 20-year secondary forest remnant was used as a reference site. Ten pitfall traps were used for the sampling of epigeous fauna at each one of the five areas (MOLDENKE, 1994). Sampling was performe in May 2000, June 2006, and April 2008. Six years after revegetation has started, fauna group richness and diversity increased both into the gully and in Mimosa caesalpiniifolia plantation. For the 2000 and 2006 samplings a PCA showed that groups were mostly associated to 2006, and completely opposite to 2000. Acacia mangium plantation had the lowest group richness. In the 2008 sampling, pasture showed the lowest group richness, and according to the PCA groups into the gully were associated to 2008, and completely opposite to 2000 and 2006. Formicidae and Collembola (Entomobryomorpha) were dominant in all sampled areas, both in 2000 and 2008, and into the gully in 2006 as well.

Keywords: recovery of degraded areas; leguminous tree; epigeal fauna.

\section{INTRODUÇÃO}

O município de Pinheiral, como toda região do Médio Vale do Paraíba do Sul, teve como primeira forma de uso de suas terras o extrativismo de madeira, posteriormente substituído, no período colonial, pela cafeicultura que, com sua decadência, deu lugar à pecuária extensiva, predominante até os dias atuais (MENEZES, 1999). Essa região teve a degradação de suas terras, causada principalmente por erosões, seja pelo carreamento de partículas orgânicas e minerais, assim como de nutrientes para o leito dos rios e para as partes baixas da paisagem. A retirada da vegetação primária para inclusão da agricultura e pecuária foram os principais fatores que levaram os solos à degradação, causando um impacto na fauna e flora. Para Machado (2007), dentre as diversas formas de erosões, as voçorocas são as que causam os maiores danos, pois carreiam nutrientes até a parte baixa e corpos d'água causando o empobrecimento das terras de forma acelerada, promovem o assoreamento dos rios e posterior inundação de casas próximas às margens, além de diminuírem a área utilizável para agricultura e pecuária.

A recuperação dessas áreas degradadas com leguminosas arbóreas tem grande vantagem em relação às demais árvores pelo fato de fixarem nitrogênio atmosférico, conseguindo se estabelecer e crescer rapidamente em solos muito pobres em fertilidade e com baixíssimo teor de matéria orgânica. Para Faria et al. (1998), algumas espécies de leguminosas que se associam a organismos diazotróficos fixadores de $\mathrm{N}$ atmosférico possuem potencial indiscutível no que diz respeito ao retorno de matéria orgânica ao solo. Por isso, assumem papel principal em um modelo tecnológico baseado na utilização daquelas espécies arbóreas cuja rusticidade e elevada produção e aporte de biomassa, represente não apenas uma proteção ao solo contra o impacto da chuva, mas um fator decisivo na aceleração da ciclagem de nutrientes e retorno da vida ao solo.

Dentre os organismos bioindicadores, os insetos são considerados muito importantes na ecologia dos ecossistemas naturais podendo ser utilizados em estudos de perturbação ambiental (ROSENBERG et al., 1986). Estes também são excelentes organismos para avaliar o impacto da formação de fragmentos florestais, pois são altamente influenciados pela heterogeneidade do habitat (THOMANZINI; THOMANZINI, 2000). Os estudos realizados baseados em bioindicadores visam usar os componentes vivos do ambiente em estudo como elementos-chave para avaliar as transformações e seus efeitos, e no caso da recuperação de áreas degradadas, monitorar os processos de remediação em diferentes partes da paisagem ao longo do tempo (PAOLETTI, 1999).

A fauna do solo epígea é responsável pela decomposição da matéria orgânica aportada pelas árvores, respondendo de forma diferenciada à quantidade e qualidade de material vegetal (MATTHEW; ZOU, 2002), principalmente a relação $\mathrm{C} / \mathrm{N}$, compostos polifenoicos e lignina. A quantidade de material vegetal também influencia na dinâmica desses organismos, pois, quanto maior a demanda de nutrientes, maior será o tempo que estes organismos permanecerão no sistema e irão atuar em processo de decomposição. Alterações 
em sua densidade e diversidade são observadas em ecossistemas que apresentam algum tipo de intervenção na sua cobertura vegetal. Desta forma, mudanças na abundância relativa e diversidade das espécies de invertebrados do solo constituemse em um bom indicador de mudanças no sistema (CURRY; GOOD, 1992).

O objetivo deste trabalho foi avaliar a diversidade da comunidade da fauna do solo epígea em uma voçoroca revegetada no ano de 2000 , com diferentes espécies de leguminosas arbóreas, ao longo do processo de sucessão ecológica em coletas realizadas no ano de 2000, 2006 e 2008.

\section{MATERIAL E MÉTODOS}

\section{Caracterização dos locais de amostragem}

O estudo foi desenvolvido no município de Pinheiral que apresenta relevo denominado por "mar de morros" com a altitude variando entre 360 e 720 metros e o clima é do tipo Cwa, de inverno seco e verão chuvoso. A média da temperatura anual encontra-se próxima a $22^{\circ} \mathrm{C}$ e a precipitação varia entre 1300 a $1500 \mathrm{~mm} \mathrm{ano}^{-1}$, com excedente hídrico de 100 a $150 \mathrm{~mm}$ mensais de dezembro a março, sendo verificada deficiência hídrica de julho a setembro (TOLEDO et al., 2002).

O trabalho foi realizado em uma voçoroca revegetada no ano 2000 localizada no Instituto Federal de Educação (IFRJ) campus Nilo Peçanha. $\mathrm{Na}$ revegetação das voçorocas foram utilizadas práticas físicas de contenção de encostas com bambu-gigante (Dendrocalamus giganteus Munro.) e pneus utilizados como paliçadas para funcionarem como barreira física de contenção de sedimentos e diminuição da velocidade de escoamento da água de precipitação. Outra prática física utilizada foi o ordenamento da água através de terraços construídos na parte superior da voçoroca com bacias de captação de água. Foram avaliadas as seguintes áreas: plantio de Acacia mangium Willd. (PA), plantio de Mimosa caesalpiniifolia Benth. (PM), plantio de leguminosas arbóreas no terço superior da encosta (PTS), plantio de leguminosas no interior da voçoroca (IVR2000). As leguminosas arbóreas utilizadas na revegetação foram: Acacia auriculiformis (A. Cunn. ex Benth.), Acacia angustissima (Mill.), Acacia mangium (Willd.), Albizia lebbeck (L.) Benth., Enterolobium contortisiliquum (Vell.), Mimosa caesalpiniifolia (Benth.), Mimosa bimucronata (DC.) O. Kuntze e Pseudosamanea guachapele
(Kunth) Harms. Os plantios de Acacia mangium e Mimosa caesalpiniifolia foram realizados antes da revegetação. Para efeito comparativo, também foi avaliada uma área de floresta secundária com 20 anos, próxima ao local de coleta.

\section{Coleta do material}

Na coleta dos dados foram utilizadas 10 armadilhas do tipo pitfall (MOLDENKE, 1994) por área. Este tipo de armadilha captura a fauna epígea que vive ativamente na superfície do solo associada aos diferentes tipos de materiais vegetais, colonizando a serrapilheira. A armadilha pitfall consiste de um pote de plástico de aproximadamente $9 \mathrm{~cm}$ de altura e $8 \mathrm{~cm}$ de diâmetro que fica em nível com o solo. A fim de conservar os organismos para a posterior triagem da fauna do solo foi utilizada uma solução de formaldeído a $4 \%$, uma vez que a armadilha permaneceu no campo por sete dias. Neste período foi utilizada uma cobertura em alumínio para evitar o transbordamento da amostra pela precipitação pluviométrica. Os organismos da fauna solo foram coletados e para conservá-los foi utilizado álcool a $70 \%$. A triagem do material foi realizada no Laboratório de Fauna do Solo da Embrapa Agrobiologia em nível de ordem, família ou grupo de acordo com Csiro (1991), Dindal (1990) e Gallo et al. (2002).

Foram realizadas três coletas na voçoroca revegetada no ano de 2000, uma no início da revegetação, em maio de 2000, em junho de 2006 e abril de 2008. No ano de 2008 foram coletadas amostras no entorno da voçoroca revegetada em 2000 (EVR2000).

\section{Análise de dados}

$\mathrm{Na}$ análise de dados foi calculado o índice de diversiade de Shannon para cada amostra de acordo com Odum (1983) pela seguinte fórmula: $\mathrm{H}=-\sum \mathrm{pi}^{*} \log \mathrm{pi}$, em que o pi corresponde a frequência relativa de cada grupo ou ordem coletada e também foi calculado o índice de Pielou $\mathrm{e}=\mathrm{H} / \log \mathrm{S}$, em que $\mathrm{H}$ é o índice de Shannon e $\mathrm{S}$ a riqueza total encontrada. Também foi calculada a riqueza média, que corresponde ao número médio de ordens ou grupos encontrados por armadilha e a riqueza total que é o número de grupos encontrados por área avaliada. Também foram calculados o número de indivíduos.armadilha ${ }^{-1}$. dia ${ }^{-1}$ de acordo com Correia e Oliveira (2000) e o respectivo erro 
padrão. Foram realizadas análises de variância pelo programa SISVAR versão 5.1 (FERREIRA, 2000) sendo utilizado o teste de Scott-Knott a 5\% de probabilidade para comparação de médias. Para isso, foram realizadas análises de normalidade dos dados pelo teste de Lilliefors e homogeneidade de variâncias pelo teste de Cochran pelo programa SAEG versão 9.1 (2007) para atender aos pressupostos da análise de variância. Os dados não homogênos e/ou não normais foram transformados por $\log (\mathrm{x}+1)$. Também foi realizada análise dos componentes principais (ACP) pelo programa CANOCO $^{\circledR}$ versão 4.0 (TER BRAAK; SMILAUER, 1998) para determinar o grau de correlação entre os grupos da fauna do solo e as áreas avaliadas para reduzir a dimensionalidade do conjunto de dados.

\section{RESULTADOS E DISCUSSÃO}

\section{Coleta realizada em 2000 e 2006}

$\mathrm{Na}$ coleta realizada no ano de 2000 , foi observada no plantio no terço superior (PTS), a maior abundância, com 28,11 \pm 5,53 indivíduos. armadilha $^{-1} \cdot$ dia $^{-1}\left(\right.$ ind. arm $\left.^{-1} \cdot \mathrm{d}^{-1}\right)$ com predominância de Entomobryomorpha (Collembola), o que fez com que este tratamento diferisse das demais áreas avaliadas neste ano. Como consequência da elevada dominância deste grupo, foi estimado, para esta área, o menor índice de diversidade de Shannon e equabilidade de Pielou (Tabela 1). Nas demais áreas amostradas, foi observado um padrão semelhante para o número de indivíduos, que não apresentaram, portanto, diferença significativa. $\mathrm{Na}$ amostragem realizada no ano de 2006, não houve diferença significativa do número de ind. $\mathrm{arm}^{-1} \cdot \mathrm{d}^{-1}$ entre as áreas. Quando comparados com os números de indivíduos entre os anos de 2000 e 2006, apenas o plantio no terço superior da voçoroca apresentou uma menor atividade da fauna do solo, após seis anos da primeira avaliação.

O maior valor de riqueza média do ano de 2000 foi observado na floresta secundária, na qual foram encontrados nove grupos em média, diferindo significativamente dos demais tratamentos. Valores próximos a esse foram observados em fragmentos florestais em diferentes estádios sucessionais no mesmo município (BATISTA et al., 2008), o que indica que esse valor de riqueza média da fauna epígea parece ser representativo das áreas florestais da região. No ano de 2006, a riqueza média para o plantio de Mimosa caesalpiniifolia, plantio no

TABELA 1: Avaliação da fauna do solo epígea nas coletas realizadas em 2000 e 2006 . Atividade da fauna do solo em indivíduos.armadilha ${ }^{-1}$.dia ${ }^{-1}$, riqueza média e total de grupos e índices de Shannon e Pielou. Pinheiral - RJ.

TABLE 1: Evaluation of epigeal soil fauna in in collections made in 2000 and 2006. Activity of soil fauna in ind.trap ${ }^{-1} \cdot \mathrm{d}^{-1}$, richness average and total groups and Shannon and Pielou index. Pinheiral RJ.

\begin{tabular}{lccccc}
\hline \multicolumn{2}{c}{ Ano 2000} & & & & \\
\hline & ind.arm ${ }^{-1} \cdot \mathrm{d}^{-1}$ & $\begin{array}{c}\text { Riqueza } \\
\text { média }\end{array}$ & $\begin{array}{c}\text { Riqueza } \\
\text { total }\end{array}$ & $\begin{array}{c}\text { Índice de } \\
\text { Shannon }\end{array}$ & $\begin{array}{c}\text { Índice de } \\
\text { Pielou }\end{array}$ \\
\hline Plantio de Acacia mangium & $5,78 \pm 0,39 \mathrm{Ab}$ & $6,88 \mathrm{Ab}$ & 13 & 2,39 & 0,65 \\
Plantio de Mimosa caesalpiniifolia & $7,61 \pm 1,57 \mathrm{Ab}$ & $5,88 \mathrm{Bb}$ & 12 & 1,76 & 0,49 \\
Plantio no terço superior & $28,11 \pm 5,53 \mathrm{Aa}$ & $5,50 \mathrm{Bb}$ & 15 & 0,90 & 0,23 \\
Interior da voçoroca & $7,79 \pm 2,22 \mathrm{Ab}$ & $5,13 \mathrm{Bb}$ & 11 & 1,18 & 0,34 \\
Mata & $6,14 \pm 1,07 \mathrm{Ab}$ & $9,00 \mathrm{Aa}$ & 21 & 2,84 & 0,65 \\
Ano 2006 & & & & & \\
Plantio de Acacia mangium & $11,89 \pm 4,82 \mathrm{Aa}$ & $6,78 \mathrm{Ab}$ & 18 & 1,23 & 0,30 \\
Plantio de Mimosa caesalpiniifolia & $11,00 \pm 2,82 \mathrm{Aa}$ & $10,00 \mathrm{Aa}$ & 24 & 2,94 & 0,64 \\
Plantio no terço superior & $9,46 \pm 2,02 \mathrm{Ba}$ & $9,44 \mathrm{Aa}$ & 23 & 2,88 & 0,64 \\
Interior da voçoroca & $8,82 \pm 1,60 \mathrm{Aa}$ & $9,78 \mathrm{Aa}$ & 23 & 2,77 & 0,61 \\
Mata & $10,44 \pm 3,11 \mathrm{Aa}$ & $8,89 \mathrm{Aa}$ & 25 & 2,42 & 0,52 \\
\hline
\end{tabular}

Em que: Valores seguidos por letra minúscula diferentes na mesma coluna e ano e maiúscula na mesma coluna e em anos diferentes, indicam diferenças significativas pelo teste de Scott-Knott a 5\% de probabilidade. 
terço superior da encosta e o interior da voçoroca revegetada em 2000 foram iguais aos da floresta, mostrando que houve uma acentuada colonização de diferentes grupos da fauna edáfica na voçoroca, o que pode ser explicado pela cobertura vegetal e conseqüente aporte de serrapilheira.

Pela comparação dos valores de riqueza total, observa-se que houve um incremento no número de grupos encontrados do ano de 2000 para o ano de 2006, o que demonstra um avanço no processo de desenvolvimento da cobertura vegetal, sendo esta capaz de prover através da serrapilheira, alimento e abrigo para a fauna edáfica. Os acréscimos na riqueza total variaram de 38 no plantio de Acacia mangium e $110 \%$ no interior da voçoroca. No interior da voçoroca no ano de 2000, foram coletados apenas 11 grupos e após 6 anos da intervenção, com plantio de mudas e utilização de práticas físicas, esse número passou para 23 grupos, indicando um restabelecimento dos processos biológicos neste sistema.

Os índices de diversidade utilizados
(Shannon e Pielou) evidencia a ocorrência de dominâncias ao longo do processo de colonização. Um exemplo disso foi observado no plantio de Acacia mangium em 2006, em que uma intensa colonização de Formicidae, com $82,6 \%$ do total de indivíduos, que teve um efeito negativo sobre os valores dos índices avaliados. Nas demais áreas houve grande aumento no índice de diversidade e uniformidade. Oliveira et al. (2005) estudando a diversidade de grupos da fauna do solo em áreas de mineração de bauxita revegetadas com leguminosas arbóreas verificaram que a diversidade de grupos foi baixa no início da revegetação, aumentando gradativamente o número de grupos a cada ano.

A análise de componentes principais (ACP) evidenciou a diferença existente entre os anos de 2000 e 2006, pois estavam em antagonismo total e a maioria dos grupos estava associada ao ano de 2006 (Figura 1). O eixo 1 diferenciou a Mata e o plantio de Mimosa caesalpiniifolia do plantio de Acacia mangium, plantio no terço superior e do interior da voçoroca revegetada em 2000. O plantio no terço

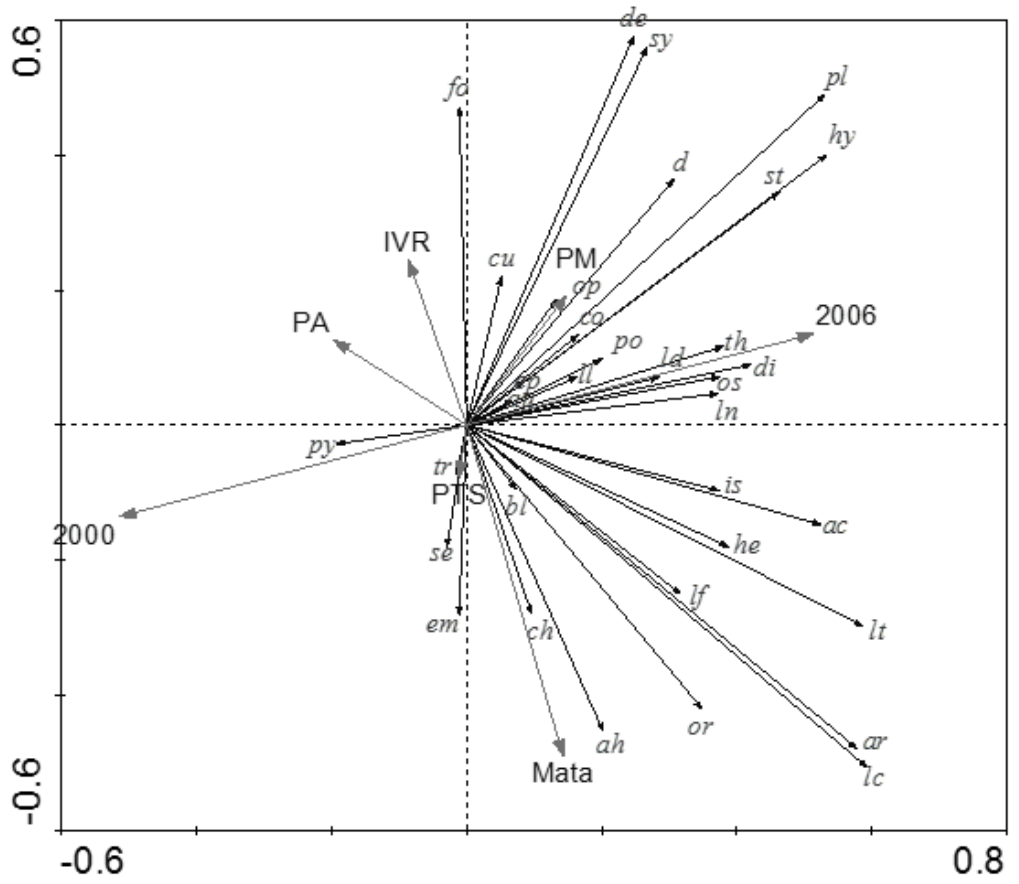

Em que: ac-Acari; ar-Araneae; au-Auchenorrhyncha; ag-Archaeognatta; bl-Blattodea; ch-Chilopoda; co-Coleoptera; de-Dermaptera; di-Diplopoda; da-Diptera; em-Entomobryomorpha; fo-Formicidae; he-Heteroptera; hy-Hymenoptera; st-Sternorrhyncha; 1t-Larvae Thicoptera; py-Polyenida; is-Isopoda; cu-Isoptera; lc- Larva de Coleoptera; ldLarva de Diptera; 11-Larva de Lepidoptera; op-Opilionida; or-Orthoptera; po-Poduromorpha; py-Polyenida; psePseudoscorpionida; pso-Psocoptera; pl-Symphypleona; th-Thysanoptera; tr-Trichoptera.

FIGURA 1: Análise de componentes principais da comunidade da fauna do solo epígea nas coletas realizadas em 2000 e 2006. Pinheiral - RJ.

FIGURE 1: Principal components analysis of epigeal soil fauna community in samples taken in 2000 and 2006. Pinheiral - RJ. 
superior apesar de baixa explicação da variabilidade, no eixo 2 juntou-se com a mata e diferenciou-se dos demais. O plantio de Acacia mangium foi a área com o menor número de grupos associados.

No ano de 2000 houve o predomínio de Collembola (Entomobryomorpha) no Plantio no terço superior e Formicidae no Interior da voçoroca revegetada em 2000, por isso foram encontrados os menores valores dos índices de diversidade para essas áreas. No ano de 2006 ocorreu o predomínio de Formicidae no plantio de Acacia mangium. Percebe-se que após 6 anos da intervenção realizada, a comunidade da fauna epígea é diferente, houve um aumento na frequência relativa dos grupos pouco numerosos no ano de 2000. Os grupos mais abundantes nos anos de 2000 e 2006 foram Formicidae e Collembola (Entomobryomorpha) e na coleta realizada em 2000 a dominância desses grupos é maior do que em 2006 (Figuras 2). Os dados obtidos por Beckmann (2009) indicam que o tempo da revegetação não influencia na recolonização das áreas por formigas, mas a cobertura vegetal foi um fator-chave para um aumento no número de espécies. Pereira et al. (2007), estudando a composição de espécies de formigas, perceberam que a estrutura da vegetação tem influência sobre a fauna edáfica. Estes autores constataram também que os ambientes reabilitados com o plantio de espécies nativas apresentam espécies diferentes quando comparadas com ambientes reabilitados com espécies exóticas e ambientes sem intervenção.

Houve um aumento da frequência relativa de Acari, Araneae, Diplopoda, Diptera, Isoptera,
Collembola (Poduromorpha) e Collembola (Symphypleona) na coleta realizada em 2006, quando comparado à primeira, apresentando maiores valores de diversidade. Percebe-se uma mudança na estrutura da comunidade da fauna do solo após seis anos da intervenção com plantio de mudas.

\section{Coleta realizada em 2008}

$\mathrm{Na}$ coleta realizada em 2008 os menores valores de atividade da fauna epígea foram observados no IVR2000 com 19,84 $\pm 3,61$ ind. arm $^{-1} \cdot \mathrm{d}^{-1}$ diferindo do EVR2000, mata secundária e pastagem que foi a área onde foram coletadas as maiores atividades da fauna do solo com 33,10 $\pm 7,06$ ind. $\mathrm{arm}^{-1} \cdot \mathrm{d}^{-1}$ (Tabela 2). No EVR2000 e Pastagem houve um predomínio de Formicidae, por isso, nestes tratamentos juntamente com a Mata foram coletados os maiores valores para atividade da fauna do solo. Toledo (2002) avaliando a fauna edáfica em duas florestas, com 20 e 60 anos de regeneração na mesma região de estudo, verificou que Formicidae, Coleoptera, Diptera, Homoptera e Hymenoptera foram os grupos mais dominantes.

Foram coletados em média 14,80 grupos na mata secundária, 13,90 no EVR 2000 e 13,70 no IVR2000 que diferiram da pastagem onde foram observados os menores valores de riqueza média. Essa diferença na riqueza média provavelmente é proveniente das leguminosas arbóreas plantadas no IVR2000 e no EVR2000 criando um microhabitat favorável para a fauna epígea, assim como ocorreu na mata secundária, não acontecendo o

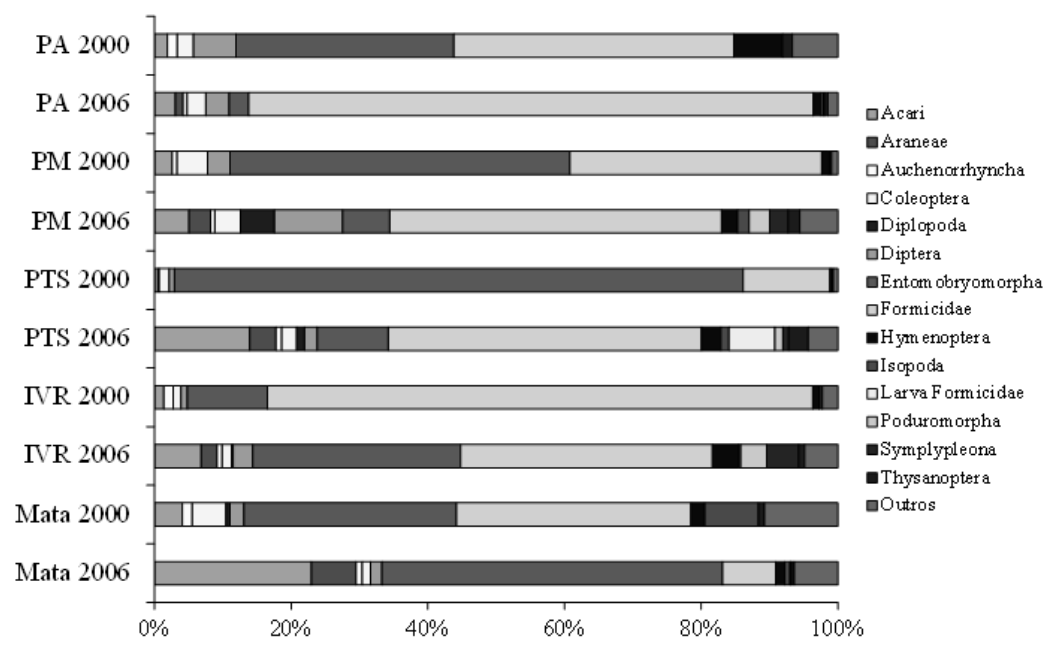

FIGURA 2: Comunidade da fauna do solo na coleta realizada no ano de 2000 e 2006. Pinheiral - RJ.

FIGURE 2: Community of soil fauna in the collection held in 2000 and 2006. Pinheiral - RJ. 
TABELA 2: Avaliação da fauna do solo epígea na coleta realizada em 2008. Atividade da fauna do solo em ind. $\mathrm{arm}^{-1} \cdot \mathrm{d}^{-1}$, riqueza média e total de grupos e índices de Shannon e Pielou. Pinheiral - RJ.

TABLE 2: Evaluation of epigeal soil fauna in the collection in 2008. Activity of soil fauna in ind.trap ${ }^{-1} \cdot \mathrm{d}^{-1}$, richness average and total groups and Shannon and Pielou index. Pinheiral - RJ.

\begin{tabular}{cccccc}
\hline \multicolumn{5}{c}{ Ano 2008 } \\
\hline & ind.arm $^{-1} \cdot \mathrm{d}^{-1}$ & Riqueza Média & Riqueza Total & Índice de Shannon & Índice de Pielou \\
\hline IVR2000 & $19,84 \pm 3,61 \mathrm{~b}$ & $13,70 \mathrm{a}$ & 24 & 2,94 & 0,64 \\
EVR2000 & $28,13 \pm 6,84 \mathrm{a}$ & $13,90 \mathrm{a}$ & 22 & 2,35 & 0,53 \\
Pastagem & $33,10 \pm 7,06 \mathrm{a}$ & $12,10 \mathrm{~b}$ & 22 & 2,25 & 0,50 \\
Mata & $23,10 \pm 3,09 \mathrm{a}$ & $14,80 \mathrm{a}$ & 25 & 3,12 & 0,67
\end{tabular}

Em que: IVR2000 - Interior da voçoroca revegetada em 2000; EVR2000 - Entorno da voçoroca revegetada em 2000. Letras minúsculas diferentes na mesma coluna indicam diferença significativa pelo teste de Scott-Knott a 5\% de probabilidade.

mesmo na área de pastagem. Não foi observada grande variação na riqueza total de grupos. Na mata secundária foram coletados 25 grupos, no IVR2000, 24. Apesar da riqueza média do EVR2000 ser maior do que a pastagem, o mesmo não foi observado na riqueza total.

O índice de diversidade de Shannon e de equabilidade de Pielou mostrou que a área menos diversa foi a pastagem, seguido pelo EVR2000, IVR2000, sendo os maiores valores observados na mata secundária. A mata apresentou os menores valores devido à menor dominância de Formicidae e Entomobryomorpha (Collembola) quando comparados com os outros tratamentos.

As maiores dominâncias foram encontradas no EVR2000 e na pastagem por Formicidae e Entomobryomorpha (Collembola) que obtiveram os menores índices de Shannon e Pielou (Figura 3). Foi encontrado um total de 30 grupos em todas as áreas. Na ACP da coleta realizada em 2008, a Mata e o IVR2000 diferiram do EVR2000 e da pastagem (Figura 4). Os grupos da fauna do solo estão associados à área de floresta secundária, ao IVR2000 e EVR2000 e na pastagem foi a área com o menor número de grupos associados.

$\mathrm{Na}$ ACP comparando o interior da voçoroca revegetada em 2000, percebe-se antagonismo total entre os anos amostrados (Figura 5). O eixo 1 diferenciou os anos de 2000 e 2006 do ano de 2008. Depois de 8 anos de revegetação os grupos da fauna

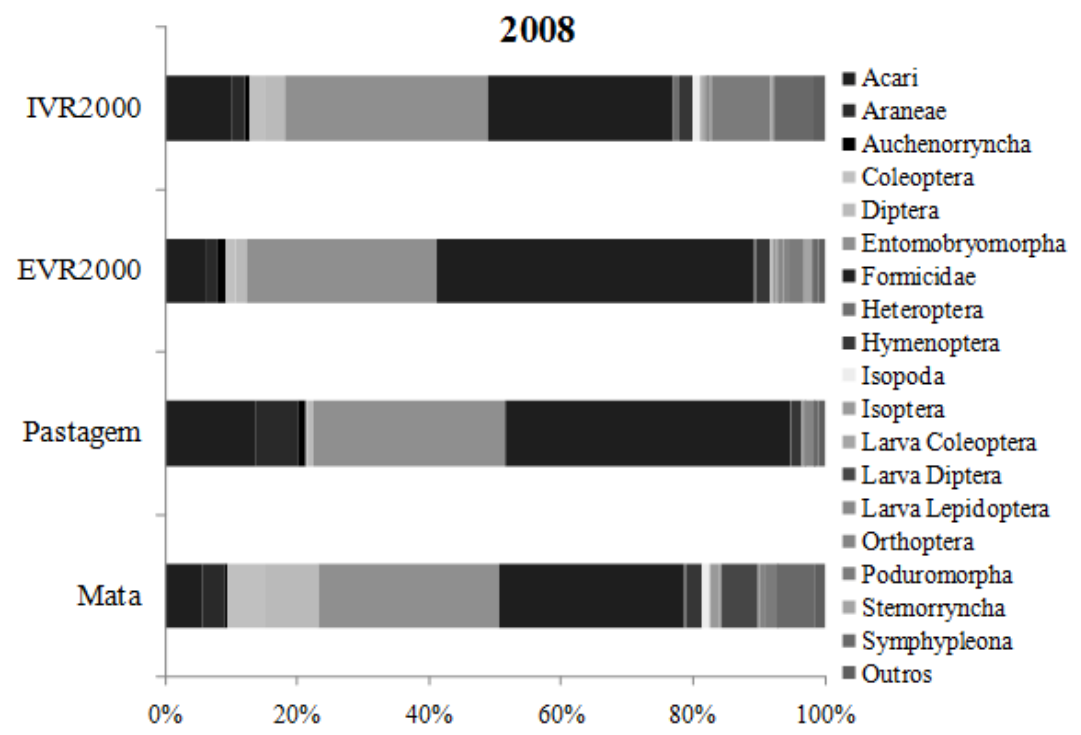

FIGURA 3: Comunidade da fauna do solo na coleta realizada no ano de 2008. Pinheiral - RJ.

FIGURE 3: Community of soil fauna in the collection held in 2008. Pinheiral - RJ. 


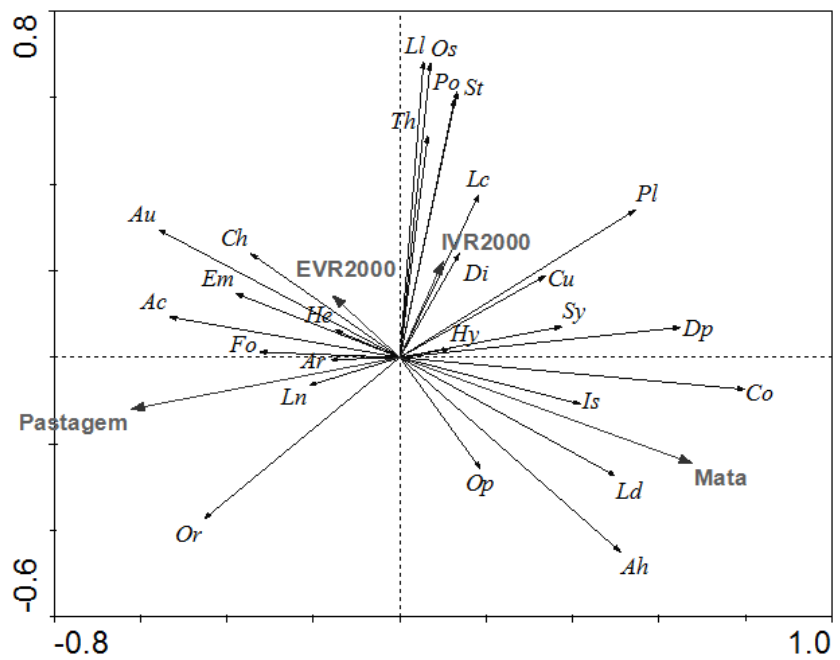

Em que: Ac-Acari; Ar-Araneae; Ah-Archaeognatha; Au-Auchenorrhyncha; B-Blattodea, Ch-Chilopoda CoColeoptera; De-Dermaptera; Di-Diplopoda; Du-Diplura, Dp-Diptera; En-Entomobryomorpha; Fo-Formicidae; He-Heteroptera; Hy-Hymenoptera; Is-Isopoda; Cu- Isoptera; Lc- Larva Coleoptera; Ld- Larva Diptera; Ll- Larva Lepidoptera; Ln-Larva Neuroptera; Lf- Larva Formicidae; Op-Opilionida; Or-Orthoptera; Po-Poduromorpha; PsPsocoptera; St- Sternorhyncha; Sy-Symphyla; Pl-Symphypleona; Ty-Thysanoptera.

FIGURA 4: Análise de componentes principais da comunidade de fauna do solo na coleta realizada em 2008. Pinheiral - RJ.

FIGURE 4: Principal components analysis of soil fauna community in the collection in 2008. Pinheiral RJ.

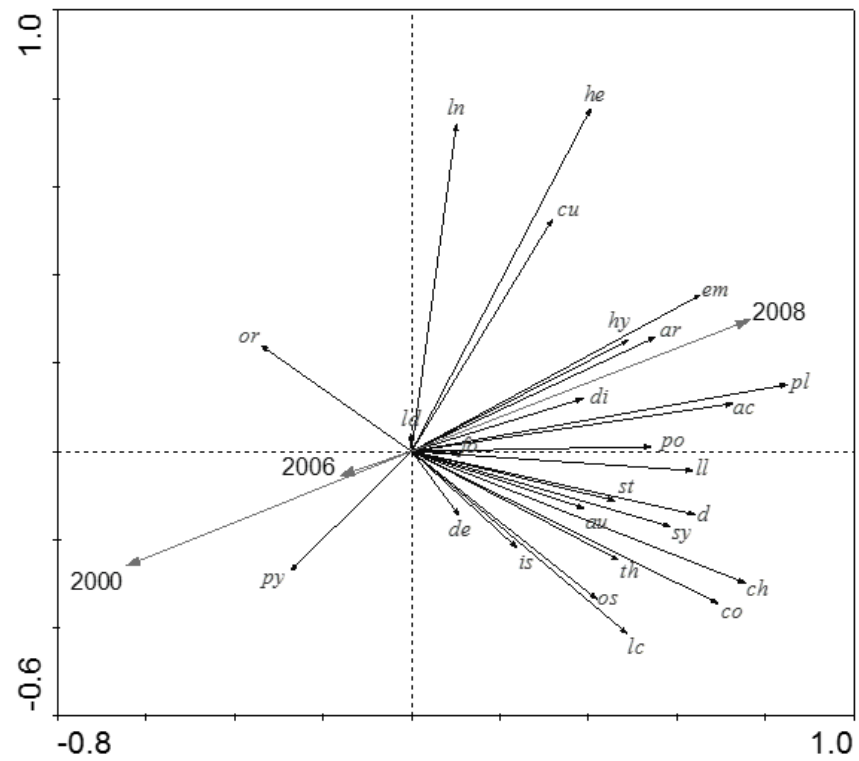

Em que: Ar-Acari; ar-Araneae; au- Auchenorrhyncha; ch-Chilopoda; co-Coleoptera; de-Dermaptera; di-Diplopoda; d-Diptera; en-Entomobryomorpha; fo-Formicidae; he-Heteroptera; hy- Hymenoptera; is-Isopoda; cu- Isoptera; lcLarva Coleoptera; ld- Larva Diptera; 11- Larva Lepidoptera; In-Larva Neuroptera; or-Orthoptera; po-Poduromorpha; py-Polyenida; os-Psocoptera; st- Sternorhyncha; sy-Symphyla; pl-Symphypleona; ty-Thysanoptera.

FIGURA 5: Análise de componentes principais da comunidade de fauna do solo no interior da voçoroca revegetada em 2000 nas três épocas amostradas. Pinheiral - RJ. Eixo 1 = 24,7\%; Eixo $2=9,90 \%$.

FIGURE 5: Principal components analysis of the community of soil fauna within the gully revegetated in 2000 the three samples collected. Pinheiral - RJ. Axis $1=24,7 \%$; Axis $2=9,90 \%$. 
do solo estavam mais abundantes no ano de 2008 devido, possivelmente, à maior oferta de alimento e micro-habitat e menos abundantes em 2006 e 2000. $\mathrm{O}$ ano de 2006 apesar de possuir a mesma tendência do ano de 2000, obteve menor grau de explicação da variabilidade. Os dados nos induzem a acreditar que à medida que a sucessão vegetal avança, com base no maior tempo de revegetação da área, condicionando o ambiente, avança também de forma sucessional, a fauna do solo.

\section{CONCLUSÕES}

Houve um aumento no número de grupos e na diversidade da fauna do solo no plantio no terço superior e no interior da voçoroca, e no plantio de Mimosa caesalpiniifolia após 6 anos da intervenção.

Nos anos de 2000 e 2006, o plantio de Acacia mangium foi o tratamento com o menor número de grupos encontrados e a pastagem no ano de 2008.

No interior da voçoroca, os grupos da fauna do solo foram responsivos ao tempo da revegetação da voçoroca.

\section{AGRADECIMENTOS}

Os autores agradecem à Embrapa Agrobiologia pelo suporte técnico para a realização deste trabalho e aos técnicos do laboratório de Leguminosas Florestais, Carlos Fernando Cunha e Telmo Felix da Silva e ao técnico do laboratório de Fauna do Solo, Roberto Silva de Oliveira.

\section{REFERÊNCIAS BIBLIOGRÁFICAS}

BATISTA, I. et al. Avaliação da fauna epígea sob diferentes sucessões florestais e pasto misto manejado em Pinheiral-RJ em época chuvosa. In: JORNADA DE INICIAÇÃO CIENTÍFICA NA UNIVERSIDADE FEDERAL RURAL DO RIO DE JANEIRO, 17., 2007, Seropédica/RS. Anais... Seropédica: EDUR, 2007. CD-ROM.

CORREIA, M. E. F.; OLIVEIRA, L. C. M. Fauna de solo: aspectos gerais e metodológicos. Seropédica: Embrapa Agrobiologia, 2000. (Embrapa Agrobiologia. Documentos, 112).

CSIRO. The Insects of Australia: a textbook for students and research workers. Carlton: Melbourne University, 1991. v. 1-2.

DINDAL, D. L. Soil biology guide. New York: Wiley, 1990.
FARIA, S. M. et al. Recuperação de Solos Degradados com Leguminosas Noduladas e Micorrizadas. Seropédica: Embrapa Agrobiologia,1998. (Embrapa-CNPAB. Documentos, 77).

FERREIRA, D. F. Análises estatísticas por meio do Sisvar para Windows versão 4.0. In: REUNIÃO ANUAL DA REGIÃO BRASILEIRA DA SOCIEDADE INTERNACIONAL DE BIOMETRIA, 45., 2000, São Carlos. Anais... São Carlos: UFSCar, 2000. p. 255-258.

GALLO, D. et al. Entomologia agrícola. 3. ed. Piracicaba: FEALQ, 2002.

MACHADO, R. L. Perda de solo e nutrientes em voçorocas com diferentes níveis de controle e recuperação no Médio Vale do rio Paraíba do Sul, RJ. 2007. 86 f. Dissertação (Mestrado em Agronomia, Ciência do Solo) - Universidade Federal Rural do Rio de Janeiro, Seropédica, 2007. MATTHEW, W.; ZOU, X. Soil macrofauna and litter nutrients in three tropical tree plantations on a disturbed site in Puerto Rico. Forest Ecology and Management, v. 170, p. 161-171, 2002.

MENEZES, C. E. G. Diagnóstico de degradação do solo em função da topografia e cobertura vegetal no município de Pinheiral, RJ. 1999. 186 p. Dissertação (Mestrado em Agronomia, Ciência do Solo) - Universidade Federal Rural do Rio de Janeiro, Seropédica, 1999.

MOLDENKE, A. R. Arthropods. In: WeAVER, R.W. et al. (Eds.). Methods of soil analysis: microbiological and biochemical properties. Part 2. Madison: SSSA, 1994. p. 517-542.

ODUM, E. P. Ecologia. Rio de Janeiro: Guanabara, 1983.

OLIVEIRA, E. P. et al. A biodiversidade da mesofauna do solo em FLONA da mineração Rio do Norte - Pará. In: SIMPÓSIO DE ÁREAS PROTEGIDAS, 3., 2005, Pelotas. Anais... Pelotas: Editora Universitária de Pelotas, 2005. CD ROM.

PAOLETTI, M. G. Using bioindicators based on biodiversity to assess landscape sustaintability. Agriculture, Ecosystems and Environment, v. 74, p. 1-18, 1999.

PEREIRA, M. P. et al. Fauna de formigas como ferramenta para monitoramento de área de mineração reabilitada na Ilha da Madeira, Itaguaí, RJ. Ciência Florestal, Santa Maria, v. 17, n. 3, p. 197-204, 2007.

ROSENBERG, D. M. et al. Importance of insects in environmental impact assessment. Environmental Management, v. 10, n. 6, p. 773-783, 1986. 
SAEG. Sistema para Análises Estatísticas, Versão 9.1. Viçosa: Fundação Arthur Bernardes, 2007. TER BRAAK, C. J. F.; SMILAUER, P. CANOCO reference manual and user's guide to Canoco for Windows: Software for canonical community ordination (version 4). New York: Microcomputer Power, 1998

TOLEDO, L. O. et al. Produção de serapilheira e transferência de nutrientes em florestas secundárias localizadas na região de Pinheiral, RJ. Ciência Florestal, Santa Maria, v. 12, n. 2, p. 9-16, 2002.

- Aporte de serrapilheira, taxa de decomposição e fauna edáfica em áreas de floresta secundária, no município de Pinheiral, RJ. 2002. 80 f. Dissertação (Mestrado em Ciências Ambientais e Florestais) - Universidade Federal Rural do Rio de Janeiro, Seropédica, 2002.

THOMANZINI, M. J.; THOMANZINI, A. P. B. W. A fragmentação florestal e a diversidade de insetos nas florestas tropicais úmidas. Rio Branco: EMBRAPA Acre, 2000. (Circular Técnica, 57). 